\title{
Developing nutrient profile models: a systematic approach
}

\author{
Peter Scarborough*, Mike Rayner and Lynn Stockley \\ British Heart Foundation Health Promotion Research Group, Department of Public Health, \\ Old Road Campus, Headington, Oxford OX3 7LF, UK
}

Submitted 21 November 2005: Accepted 8 March 2006

\begin{abstract}
Objective: Nutrient profiling can be defined as the 'the science of categorising foods according to their nutritional composition'. The purpose of the present paper is to describe a systematic and logical approach to nutrient profiling.

Design: A seven-stage decision-making process is proposed and, as an illustration of how the approach might operate in practice, the development of a nutrient profiling model for the purpose of highlighting breakfast cereals that are 'high in fat, sugar or salt' is described.

Results: The nutrient profile model developed for this paper calculates scores for foods using a simple equation. It enables breakfast cereals to be compared with each other and with other foods eaten at breakfast.

Conclusion: Nutrient profiling is not new, but hitherto most nutrient profiling models have been developed in an unsystematic and illogical fashion. Different nutrient profiling models are needed for different purposes but a key requirement should be that they are developed using a systematic, transparent and logical process. This paper provides an example of such a process; approaches to validating nutrient profiling models are described elsewhere.
\end{abstract}

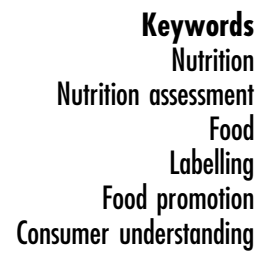

Unhealthy diets lead to an increased risk of coronary heart disease, stroke, some forms of cancer and a range of other diseases ${ }^{1}$. A healthy diet consists of a variety of different foods, but some foods are more likely to be constituents of a healthy diet than others. Various ways of identifying foods that are more or less likely to be constituents of healthy and unhealthy diets have been identified $^{2}$. One way is to use nutrient profiling: a term first coined but not defined in a proposal for the regulation of health and nutrition claims published by the European Commission ${ }^{3}$.

Nutrient profiling can be defined as the science of categorising foods according to their nutritional composition'4. It is useful in a range of different circumstances including food labelling and its regulation, the regulation of food marketing, etc. ${ }^{3,5}$. The alternative to using nutritional composition to categorise foods is to use nonnutritional features such as their biological characteristics (e.g. the descriptors 'fruit', 'offal'), their means of production (e.g. 'biscuit', 'yoghurt'), etc. The problem with using such features for health-related purposes is that they often bear little relation to the health-related properties of the foods; for example, yoghurts may or may not be high in added sugar.

There are several circumstances where nutrient profiling has been used in the past without being described as such: for example, the proposed European
Union (EU) regulation on nutrition and health claims contains compositional criteria for simple nutrition claims such as 'low in fat', 'high in fibre', etc. Some health charities and other bodies - including government agencies - have developed criteria for symbols which food producers can use to label foods as 'healthy'. A recent review summarises such initiatives'. In general the criteria have been developed from previous criteria on an ad hoc basis. Here we propose a more systematic, transparent and logical process for developing criteria.

The approach that we describe here has been used in work for the Food Standards Agency (FSA) in the UK to develop and agree a model with associated definitions of 'healthier' and 'less healthy' foods in relation to the promotion of food to children ${ }^{4,6,7}$, but we propose that it could - with care and consideration - be used generally for nutrient profiling. In the present paper we show how the approach might be used to develop a definition for a food that is 'high in fat, sugar or salt' (a relatively simple descriptor) as an illustration of the value of the approach.

The proposed approach consists of seven stages. These stages are presented as sequential but decisions made at some stages can affect decisions made at previous stages, so decision-making therefore needs to be iterative. The stages we propose involve asking the following questions: 
- For what purpose is the model to be used?

- What group or population is the purpose relevant to?

- Are food-category-specific or across-the-board criteria more appropriate for the purpose?

- Which nutrients or other food components should be included?

- Which base or combination of bases (e.g. per $100 \mathrm{~g}$, per serving or per $100 \mathrm{~kJ}$ ) should be used?

- What type of model should be used (e.g. one using a 'threshold' for nutrient/food criteria or one which allocates 'scores' to nutrient/food criteria)?

- What numbers should be used for the criteria?

One of the reports from the FSA reporting the development of a model with associated definitions of 'healthier' and 'less healthy' foods in relation to the promotion of food to children describes the choices available at some of the stages of this approach in more detail than is possible here ${ }^{4}$.

\section{Methods for developing a nutrient profile model}

\section{Stage 1: Deciding for what purpose the model is to be used}

Criteria for descriptions of foods such as 'low in fat', 'less healthy', etc. may need to be different depending on the purpose for which they are required. Sometimes simple definitions will be necessary; for example, if they are to be used as rules of thumb by a consumer trying to interpret the nutrition labelling panel on a food packet. In other cases they may need to be complex; for example, where the definitions are to be used by health professionals or the food industry to categorise foods and where a wide range of nutrients and other food components are involved.

The descriptor 'high in fat', sugar or salt' is commonly used as a synonym for 'a less healthy food' and there are many purposes for which a clear definition of a 'less healthy food' would be useful. However, not all foods that might be defined as 'high in fat, sugar or salt' would necessarily be defined as 'less healthy foods'; for example, fruit is high in sugar. So definitions of 'less healthy foods' need to take account of other characteristics of the food: whether the sugars are intrinsic or extrinsic sugars, the levels of minerals and vitamins, etc.

In this paper we consider the development of a simple model to highlight foods which are 'high in fat, sugar or salt', regardless of their other characteristics, for the purpose of a theoretical magazine article dealing with the health benefits of different breakfast cereals. The development of this model will reflect this purpose, and the choices made at each stage of the process may not be relevant for other purposes (such as a system for simplifying nutrition labelling).

\section{Stage 2: Deciding what group or population the purpose is relevant to}

Descriptors such as 'low in fat', 'healthy', etc. need different definitions depending on the age and other characteristics of the population for whom the purpose is relevant. For example, breast milk is 'healthy' for a newborn baby but it contains a level of fat that is not optimal and therefore not 'healthy' for an adult. The same argument applies when drawing up definitions of descriptors such as 'low in fat' or 'healthy' for other specific subgroups of the population such as adolescents, women planning pregnancy and older people.

In developing a nutrient profile model for 'high in fat, sugar or salt' for use in an article about breakfast cereals, we imagined that the target group would be the general adult population.

\section{Stage 3: Deciding whether to use food-category- specific or across-tbe-board criteria}

Descriptors defined by nutrient profile models can be absolute, or relative to other foods. In regulating nutrition claims such as 'low in fat' it has come to be assumed that such claims mean that the food is low in fat relative to all other foods - and not just similar foods or foods of the same food category. For example, the European Commission currently proposes that the criterion for the claim 'low in fat' should be that the food has less than $3 \mathrm{~g}$ of fat per $100 \mathrm{~g}$ of food regardless of the type of food (i.e. 'acrossthe-board'). Conversely, in regulating the claim 'lower in fat' or 'reduced fat', it has come to be assumed that such claims mean that the food is low in fat relative to foods of the same category (i.e. 'category-specific') ${ }^{3}$.

With criteria for 'healthy' and 'healthier' - and their synonyms - there is less agreement and a good deal of confusion. Most of the schemes that have been developed by public health organisations and the food industry to label foods as 'healthy' or 'healthier' tend to use food-category-specific criteria. One of the best known of such schemes is the Tick Programme run by the National Heart Foundation of Australia. This has different criteria for over 60 categories of foods ${ }^{8}$. The symbol sometimes signifies 'healthy' per se and sometimes 'healthier' than other foods within a category. This may not be clear to consumers. There are, on the other hand, a few nutrient profiling models which use across-the-board criteria to classify foods as 'healthy' or 'healthier'9-11. The definitions of 'healthier' and of 'less healthy' foods that were developed for use in relation to regulating the promotion of food to children involve across-the-board criteria ${ }^{4}$.

If a food labelling scheme is designed to encourage consumers to switch to healthier foods within a food category, then category-specific criteria are appropriate. But if it is designed to encourage consumers to switch to healthier food categories, for example from confectionery to fruit, across-the-board criteria will be necessary.

If category-specific criteria are required then food categories need to be defined, which is far from straightforward. There are no universally accepted food categories and many different systems are used even 
under similar circumstances (e.g. the Australian Tick Programme $^{8}$ and the Swedish Green Keyhole ${ }^{12}$ scheme are similar category-specific labelling schemes but use very different food categories). In addition, new composite foods involving foods from more than one category are constantly being developed.

In developing a nutrient profile model for 'high in fat, sugar or salt', for use in an article about breakfast cereals, across-the-board criteria are probably the most appropriate. This is because it is now conventional to define nutrition claims such as 'low in fat' or 'low in sugar' as low in those nutrients relative to all foods. For a definition of 'higher in fat, sugar or salt' it would probably be more appropriate to use category-specific criteria, since the term 'higher' implies a comparison with similar foods.

Furthermore, in developing a nutrient profile model for 'high in fat, sugar or salt' we considered that it would be useful for consumers to be able to compare breakfast cereals with other foods eaten at breakfast; for example, toast, croissant, bacon and fruit. Using across-the-board criteria allows this.

\section{Stage 4: Deciding which nutrients and otber food components to use}

Nutrient profiling models can sometimes involve components which technically are not nutrients, such as energy or water, and even ingredients such as 'fruit and vegetables', or food additives such as preservatives. Hereafter for simplicity we will call all the possible components of nutrient profiling 'nutrients'.

The nutrients selected for nutrient profile models depend on the definition required. The choice is clear when the descriptor is 'low/lower in $x$ ' or even 'low/lower in $x, y$ or $z$ ' where $x, y$ and $z$ are named nutrients. It is less clear when the descriptor is 'healthy' or 'less healthy'. In that case, the number of different combinations of nutrients and food components that could possibly be used for nutrient profiling is considerable. A recent World Health Organization report lists 37 nutrients and other food components linked with chronic disease ${ }^{1}$. The EU directive on nutrition labelling lists 31 nutrients which it is permitted to include within the nutrition labelling panel ${ }^{13}$.

Nutrient profiling necessarily involves prioritisation of nutrients. In framing the EU legislation for nutrition labelling, the legislators chose four nutrients that must be presented if nutrition labelling is provided: energy, protein, fat and carbohydrate. These nutrients, in effect, represent the 'priority nutrients' that the legislators considered were most important for consumers to know about for health-related purposes.

Nutrients are normally prioritised because levels in the population or population subgroup are important for public health. Thus most definitions of 'healthy' or 'healthier' involve criteria for fat, saturated fat and sodium. But nutrients may also be prioritised because they are markers of foods, the consumption of which is considered to be important for public health. So, for example, in developing definitions of 'less healthy' and 'healthier' food for the purpose of regulating the promotion of food to children ${ }^{4}$ it was agreed that the 'healthier' category should have a significant number of foods from the 'meat and alternatives' group of the UK's national food guide (The Balance of Good Health ${ }^{14}$ ); initially iron and subsequently protein were incorporated into the models to ensure this.

In selecting nutrients for a nutrient profiling model there will also be practical considerations that need to be taken into account - e.g. for food labelling purposes definitions of 'healthy' or 'healthier' may need to be verifiable by analysis, limiting the choice of nutrients to those which can be measured in a laboratory.

The choice of nutrients for a definition for 'high in fat, sugar or salt' would - on the face of it - seem largely predetermined. However, even here there are some remaining choices to be made. In developing the model we have chosen to use sodium levels rather than salt levels because it is the sodium in salt which is of public health concern. We have also opted for non-milk extrinsic sugars (NMES) since these are the subject of officially agreed population dietary goals rather than added sugar or total sugar $^{15}$.

\section{Stage 5: Deciding which base or combination of bases to use}

Theoretically, the amount of nutrient in a food can be expressed in many different ways (bases). However, in practice, only per $100 \mathrm{~g}$, per $100 \mathrm{~kJ}$ or per serving, or combinations of these, are practical. There are advantages and disadvantages to all three of these bases.

'Per $100 \mathrm{~g}$ ' is the base used most often for nutrient profiling, probably because this is the base used in food composition tables and in the required format for nutrition labelling in the EU. However, a food that is high in a nutrient on a 'per $100 \mathrm{~g}$ ' basis may supply little of that nutrient to the diet because it is eaten in small amounts and/or infrequently (e.g. fat in mustard). Conversely, a food that may be low in a nutrient 'per $100 \mathrm{~g}$ ' may supply a lot of that nutrient to the diet because it is eaten in large amounts and/or frequently (e.g. NMES in soft drinks). One of the main reasons for this is that foods vary widely in their water content and foods that contain high levels of water tend to be eaten in large amounts (e.g. beverages, stews, etc.). Of course, there are other determinants of amount and/or frequency of consumption besides the water content of foods, such as intensity of taste (e.g. yeast extract).

Using a 'per $100 \mathrm{~kJ}$ ' basis means that the water content of foods is discounted. The amount of food that people eat is, in general, governed by their energy needs (weight-stable individuals will consume enough food to satisfy their energy requirements; those seeking to lose weight will generally aim to consume less food in order to reduce their energy intake; and those increasing in weight will normally be consuming slightly more food than they 
need to supply them with energy). One of the consequences of this is that there is stronger correlation between nutrient 'per serving' and nutrient 'per $100 \mathrm{~kJ}$ ' than between nutrient 'per serving' and nutrient 'per 100 g.* Using an energy basis therefore partially takes account of the amount of food people generally eat.

However, there are still some energy-dense foods such as mustard - which appear high in a nutrient when measured 'per $100 \mathrm{~kJ}$ ', yet supply little of that nutrient to the diet because they are eaten in small servings and/or infrequently. Foods with very high water content, such as some fruits and vegetables, pose a further problem. These foods tend to supply very little energy and so can appear high in a nutrient on a 'per $100 \mathrm{~kJ}$ basis' (e.g. raw celery contains $200 \mathrm{mg}$ of sodium 'per $100 \mathrm{~kJ}$ ', enough to be categorised as 'high sodium' by schemes which use a 'per $100 \mathrm{~kJ}^{\prime}$ basis $^{5}$ ).

The use of a 'per serving' basis for nutrient profiles also partially takes account of the way people eat foods because serving size, together with frequency of consumption, are the main determinants of how much of a nutrient a food supplies to the diet. However, serving size for many foods varies considerably. For example, the serving size of milk depends on whether it is drunk by itself or with breakfast cereal or with coffee. Also, serving sizes partly depend on the energy needs of the consumer (e.g. young children tend to eat smaller serving sizes than adults). Serving sizes for some foods are therefore difficult to define. In addition, for some foods the serving size bears little relation to how much of a nutrient it contributes to the diet. For example, the serving size for margarine is small, but servings of margarine may be eaten on many occasions in a single day and so may contribute a substantial amount of fat to a diet.

The choice of which base is used for nutrient profiles is crucial, as different bases will rank foods in different orders (e.g. spaghetti bolognaise has less fat 'per 100 g' than mayonnaise, but more fat 'per serving'). However, the choice of which base to use becomes less important if 'food-category-specific' criteria are used because foods within categories tend to vary less in their water content or serving size than the whole range of foods.

In developing nutrient profile models for categorising foods there has been considerable confusion over which base to use. The proposed EU regulation on health and nutrition claims sets criteria for nutrition claims on a 'per $100 \mathrm{~g}$ ' basis $^{3}$ whereas the US Nutrition Labelling and Education Act sets criteria for nutrition claims on a per serving' basis ${ }^{17}$. There are some - albeit rare - examples of the use of 'per $100 \mathrm{~kJ}$ ' base'.

*Using a database of foods derived from McCance and Widdowson's The Composition of Foods ${ }^{16}$, the correlation $\left(r^{2}\right)$ between fat levels measured per $100 \mathrm{~g}$ and per $100 \mathrm{~kJ}$ was 0.63 , whereas the correlation between the amount of fat per serving and the amount of fat per $100 \mathrm{~kJ}$ was 0.34 , and that between the amount of fat per serving and the amount of fat per $100 \mathrm{~g}$ was 0.20 .
It is possible to combine two bases for nutrient profiles so as to gain the advantages of both bases, and this can and has been used for nutrient profiling. For example, the Codex Alimentarius definition for 'low in saturated fat' is that a food should have less than $1.5 \mathrm{~g}$ of saturated fat 'per $100 \mathrm{~g}$ ' and less than $10 \mathrm{~kJ}$ of saturated fat 'per $100 \mathrm{~kJ}$ '18.

In developing a nutrient profile model for 'high in fat, sugar or salt' we decided that it would be most useful if it helped people compare the quantity of fat, salt and sugar supplied to the diet by different foods, rather than compare the nutritional quality of these foods. This is best achieved using a 'per serving' base.

\section{Stage 6: Deciding which model type to use}

Nutrient profile models vary considerably in their design but fundamentally they adopt one of two approaches: categorical or continuous. Categorical models divide foods into two or more categories but beyond this categorisation foods can no longer be compared (e.g. a categorical model may categorise two foods as high in salt', but will not indicate which food contains more salt). Continuous models provide a ranking of foods and so are intrinsically more complex than categorical models and therefore impractical for some purposes.

Categorical models are also called threshold models because they operate by considering whether or not a food has a nutrient content higher or lower than a specified threshold or thresholds. Categorical models are the most common type of nutrient profile model. Clearly criteria for nutrition claims such as 'low in fat' have to be categorical. The criteria for the schemes that have been developed by public health organisations and the food industry to label foods as 'healthy' or 'healthier' tend to be categorical models where foods are categorised according to whether the nutrient levels are higher or lower than a number of specified thresholds.

Continuous models are often called scoring systems, as they operate by calculating a score for each food which can then be used to rank foods. Continuous models can be converted into categorical models simply by setting score threshold criteria. For example, a food might be defined as 'healthy' if it scores a certain number of points or more. Continuous models are more flexible than categorical models since different score thresholds can be set for different food categories or for different purposes.

With continuous models any small change in the nutritional value of a food will result in a small change to the score for that food. There are several examples of such continuous models ${ }^{9-11}$. The nutrient profile model developed for use in relation to regulating the promotion of food to children ${ }^{4}$ is a semi-continuous model. For this model the final score for a food is always a whole number, and is determined by considering a number of score bands for each nutrient. Hence, the scores are categorical and a small change in the nutritional value of a food may not result in a change to the score of the food. However, the 
other advantages of continuous models (flexibility, direct comparability) are retained.

The primary advantage of continuous models over categorical models is their ability to differentiate between foods that are high in a number of nutrients and foods that are high in only one nutrient. For example, two biscuits one of which is high in saturated fat and high in sodium, the other high in saturated fat but low in sodium - are indistinguishable using a categorical model used to define foods that are high in saturated fat or sodium. However, a continuous model used for the same definition would rank the two biscuits appropriately, thereby providing more information to the user of the model.

In developing a nutrient profile model for 'high in fat, sugar or salt' we have chosen to use a continuous approach so that the model will provide a ranking of foods.

\section{Stage 7: Choosing the numbers to use}

The levels set for the thresholds in categorical models or the numbers of points scored for a particular nutrient level in continuous models can be selected in a number of ways. For example, they can be chosen pragmatically, taken from respected sources or linked to public health recommendations.

In many cases the thresholds in existing categorical models appear to have been chosen pragmatically so that certain foods are included within a definition and/or so that that the levels coincide with food producers' views of what are attainable. In other cases the thresholds are taken from other respected sources (e.g. the proposed EU definition for a 'low fat' claim is consciously taken from the Codex Alimentarius definition, which in turn is a modification of the US definition (EU and Codex: $<3 \mathrm{~g}$ per $100 \mathrm{~g}$; US: $<3 \mathrm{~g}$ per serving $\left.{ }^{3,17,18}\right)$ ). Such thresholds might be justifiable on the basis that they have been tried, and to some degree tested, elsewhere but they do not have any transparent or logical relationship to dietary recommendations.

In developing nutrient profile models it is possible to ensure that threshold levels or points scored are explicitly and logically linked to public health recommendations. This can be done in various ways. One way of doing this with across-the-board models is to use standard proportions of what have come to be known as Guideline Daily Amounts (GDAs) ${ }^{19}$. This was the approach taken in developing FSA advice on what counts as a lot or a little of various nutrients ${ }^{18}$ and in developing definitions of 'less healthy' foods for use in relation to the promotion of food to children ${ }^{4}$. Another way of linking to public health recommendations, which is particularly suitable for foodcategory-specific criteria, is by modelling what needs to be achieved to reach desirable intake levels from current intake levels ${ }^{20}$.

We had decided that the purpose of our nutrient profile model for 'high in fat, sugar or salt' was to help adults compare the nutrient content of different foods eaten at breakfast; therefore we decided that the numbers chosen for the points scored at different levels of fat, sodium and NMES should be related, in a standard way, to adult GDAs (sexes combined) of $82.3 \mathrm{~g}$ for fat, $2.3 \mathrm{~g}$ for sodium and $59.9 \mathrm{~g}$ for NMES. These GDAs were calculated in a way described previously ${ }^{19}$ and are similar to GDAs recently proposed by the Institute of Grocery Distribution ${ }^{21}$.

\section{Results of developing a nutrient profile model for comparing breakfast cereals}

Using the seven-stage decision-making process to nutrient profiling that we have outlined above generates a simple nutrient profiling model for the purpose of highlighting breakfast cereals that are 'high in fat, sugar or salt'. The model has the following equation:

$$
\begin{aligned}
\text { Score }= & \frac{\text { Total fat }(\mathrm{g} \text { per serving })}{\text { Total fat GDA }}+\frac{\text { NMES }(\mathrm{g} \text { per serving })}{\text { NMES GDA }} \\
& +\frac{\text { Sodium }(\mathrm{g} \text { per serving) }}{\text { Sodium GDA }}
\end{aligned}
$$

The equation was used to calculate scores for a selection of breakfast cereals and other foods eaten at breakfast, using food composition data from the McCance and Widdowson database ${ }^{16}$ and serving size data published by the former Ministry of Agriculture, Fisheries and Food ${ }^{22}$. (The data for the NMES content were derived from the total sugars data by a method described previously ${ }^{4}$.)

The foods and their scores are presented in Table 1.

One possible definition of a food that is 'high in fat, sugar or salt' would be one that has a lot of fat, sugar or salt compared with the FSA advice on what constitutes 'a lot' 19 . This would be a simple categorical model. The data presented in Table 1 show that using this definition would mean that Sugar Puffs, Frosties, white bread toasted, wholemeal bread toasted, streaky bacon and scrambled egg count as such foods.

The continuous model developed for this paper ranks foods as in Table 1, split into 'breakfast cereals' and 'other breakfast food' categories. This continuous model can be converted to a categorical model by setting an arbitrary threshold score above which foods are defined as 'high in fat, sugar or salt'. If this threshold score is set at 325 then Frosties and streaky bacon would be defined as 'high in fat, sugar or salt', but Sugar Puffs, white bread toasted, wholemeal bread toasted and scrambled egg would not. In addition, Bran Flakes, Cheerios and Sultana Bran would be defined as 'high in fat, sugar or salt'.

In our view, the continuous model is preferable to the categorical model because it takes account of medium levels of fat, salt and sugar and not just very high levels of these nutrients when categorising the foods. But we accept that this judgement should be subject to empirical verification. Methods of validating and comparing different nutrient profiling models will be dealt with elsewhere (Scarborough P, Boxer A, Rayner M and Stockley L, Testing 
Table 1 Serving sizes and nutrient content of breakfast cereals and other foods eaten at breakfast, together with nutrient profile model scores

\begin{tabular}{|c|c|c|c|c|c|}
\hline Food & $\begin{array}{l}\text { Serving } \\
\text { size }(g)^{\star}\end{array}$ & Fat (g per $100 \mathrm{~g}$ ) & NMES (g per $100 \mathrm{~g}$ ) & $\begin{array}{c}\text { Sodium } \\
\text { (mg per } 100 \mathrm{~g})\end{array}$ & Score $(\times 1000)$ \\
\hline Puffed Wheat & 120 & 1.6 & 0.1 & 37 & 44 \\
\hline Shredded Wheat & 145 & 1.8 & 0.2 & 32 & 57 \\
\hline Weetabix & 140 & 2.0 & 1.4 & 108 & 132 \\
\hline Rice Krispies & 130 & 1.5 & 2.4 & 183 & 180 \\
\hline Corn Flakes & 130 & 1.5 & 1.9 & 264 & 214 \\
\hline Special K & 130 & 1.5 & 3.9 & 218 & 231 \\
\hline Fruit 'n Fibre & 130 & 2.5 & 5.3 & 172 & 251 \\
\hline Shreddies & 145 & 1.8 & 4.8 & 200 & 273 \\
\hline Sugar Puffs & 130 & 1.5 & 11.9 & 35 & 302 \\
\hline Ricicles & 130 & 1.5 & 9.5 & 137 & 306 \\
\hline Coco Pops & 130 & 1.9 & 9.7 & 137 & 318 \\
\hline Crunchy Nut Corn Flakes & 130 & 2.1 & 8.7 & 172 & 319 \\
\hline Frosties & 130 & 1.4 & 10.2 & 172 & 340 \\
\hline Bran Flakes & 140 & 1.9 & 6.5 & 259 & 343 \\
\hline Cheerios & 140 & 2.3 & 6.4 & 259 & 347 \\
\hline Sultana Bran & 140 & 1.8 & 9.6 & 202 & 377 \\
\hline Grapefruit, raw & 80 & 0.1 & 0.0 & 3 & 2 \\
\hline White bread, toasted & 27 & 2.0 & 4.1 & 562 & 91 \\
\hline Boiled egg & 50 & 10.8 & 0.0 & 140 & 96 \\
\hline Wholemeal bread, toasted & 31 & 2.9 & 3.2 & 570 & 104 \\
\hline Croissant & 60 & 19.7 & 5.1 & 419 & 304 \\
\hline Scrambled egg & 120 & 23.4 & 0.0 & 222 & 457 \\
\hline Bacon, streaky, fried & 46 & 26.6 & 0.0 & 681 & 525 \\
\hline
\end{tabular}

NMES - non-milk extrinsic sugars.

'High' levels of fat, NMES and sodium are indicated in bold, using the levels that the Food Standards Agency currently advises constitute 'a lot'19 ('a lot of fat', > $20 \mathrm{~g}$ per $100 \mathrm{~g}$; 'a lot of NMES', >10 g per $100 \mathrm{~g}$; 'a lot of sodium', >500 mg per $100 \mathrm{~g}$ ).

Note: The source of the nutrient composition data was the McCance and Widdowson database published in 2003 , and may no longer reflect the current nutritional composition of the products listed.

* Serving sizes for breakfast cereals include $100 \mathrm{~g}$ of semi-skimmed milk.

and validating nutrient profile models using data from a survey of nutrition professionals, submitted).

\section{Conclusions}

The results of developing a nutrient profiling model to define a food as 'high in fat, sugar or salt' illustrate the particular advantage of continuous and across-the-board models, namely that foods can be compared within and outside particular food categories. The nutrient profiling scores shown in Table 1 clearly indicate which breakfast cereals are higher in fat, sugar or salt. But they also show how individual breakfast cereals compare with other foods eaten at breakfast. They show, for example, that individuals concerned only with reducing fat, NMES and sodium levels in their diet should regard all the breakfast cereals to be a better choice than scrambled egg and bacon, and most (but not all) would be a better choice than a croissant.

The model was designed to illustrate the decisionmaking process at each of seven stages of a systematic approach to nutrient profiling, and the results should be viewed in that light. The model considers only a limited number of nutrients, and does not provide a complete picture of which foods are most likely to contribute towards a healthier breakfast. To achieve this, a more comprehensive approach would be needed, particularly in relation to the choice of nutrients and food components.
The systematic approach to nutrient profiling we propose has the capacity to generate many different nutrient profile models even for the same purpose. Having generated different models they need to be tested to ascertain whether they are appropriate to the purpose. The issue of testing and validating nutrient profile models has not been raised here, but is covered in previous publications $^{4,6}$ and a parallel paper (Scarborough P, Boxer A, Rayner M and Stockley L, Testing and validating nutrient profile models using data from a survey of nutrition professionals, submitted).

Nutrient profiling has, in the past, generally been carried out in a haphazard fashion. The approach we propose here could help all those involved in nutrient profiling to systematise their methods.

\section{Acknowledgements}

P.S. and M.R. are supported by funding provided by the British Heart Foundation. The approach described in this paper was developed while the authors were carrying out work funded by the FSA. The authors are grateful to Rosemary Hignett, Mark Browne and others from the FSA and to members of the Expert Group who oversaw the work funded by the FSA for help in clarifying the ideas contained in this paper. 


\section{References}

1 World Health Organization (WHO). Food and Health in Europe: A New Basis of Action. Copenhagen: WHO Regional Office for Europe, 2002.

2 World Health Organization (WHO). Diet, Nutrition and the Prevention of Chronic Diseases. Report of a Joint WHO/Food and Agriculture Organization Expert Consultation. WHO Technical Report Series No. 916. Geneva: WHO, 2003.

3 European Commission. Proposal for a Regulation of the European Parliament and of the Council on Nutrition and Health Claims Made on Foods. Brussels: European Commission, 16 July 2003; 2003/0165 (COD).

4 Rayner M, Scarborough P, Stockley L. Nutrient Profiles: Options for Definitions for Use in Relation to the Promotion of Foods to Children. London: Food Standards Agency, 2004. Also available at http://www.food.gov.uk/multimedia/pdfs/ nutrientprofilingfullreport.pdf

5 Stockley L. Nutrition Profiles for Foods to which Nutrients can be Added, or on which Health Claims can be Made. London: Food Standards Agency, 2003.

6 Rayner M, Scarborough P, Stockley L, Boxer A. Nutrient Profiles: Further Refinement and Testing of Model SSCg3d. London: Food Standards Agency, 2005. Also available at http://www.food.gov.uk/multimedia/pdfs/npreportsept05. pdf

7 Rayner M, Scarborough P, Boxer A, Stockley L. Nutrient Profiles: Development of Final Model. London: Food Standards Agency, 2005.

8 National Heart Foundation of Australia. Guidelines for Tick Approval [online], 2006. Available at http://www.heart foundation.com.au/index.cfm?page $=975$. Accessed 18 Sept ember 2006.

9 Gazibarich B, Ricci PF. Towards better food choices: the nutritious food index. Australian Journal of Nutrition and Dietetics 1998; 55: 10-20.

10 Scheidt DM, Daniel E. Composite index for aggregating nutrient density using food labels: ration of recommended to restricted food components. Journal of Nutrition Education and Behavior 2004; 36: 35-41.
11 Zelman K, Kennedy A. Naturally nutrient rich ... putting more power on Americans' plates. Nutrition Today 2005; 40: $60-8$.

12 National Food Association of Sweden. Statute Book of the National Food Administration. Stockholm: National Food Administration, 2004

13 Council Directive 90/496 of 24 September 1990 on nutrition labelling for foodstuffs. Official Journal 06/10/1990; L276; $40-4$.

14 Health Education Authority (HEA). The Balance of Good Health. London: HEA, 1994

15 Committee for Medical Aspects of Food Policy. Dietary Reference Values for Food Energy and Nutrients for the United Kingdom. Department of Health Report on Health and Social Subjects No. 41. London: HMSO, 1991.

16 Roe M, Finglas P, Church S. McCance \& Widdowson's The Composition of Foods, 6th summary ed. London: Royal Society of Chemistry, 2003.

17 US Food and Drug Administration. Guide to Nutrition Labeling and Education Act (NLEA) Requirements [online], 1994. Available at http://www.fda.gov/ora/inspect_ref/igs/ nleatxt.html. Accessed 18 September 2006.

18 Food and Agriculture Organization/World Health Organization Food Standards. Understanding the Codex Alimentarius [online], 2005. Available at http://www.fao.org/docrep/008/ y7867e/y7867e00.htm. Accessed 18 September 2006.

19 Rayner M, Scarborough P, Williams C. The origin of Guideline Daily Amounts and the Food Standards Agency's guidance on what counts as 'a lot' and 'a little'. Public Health Nutrition 2004; 7: 549-56.

20 Food Standards Agency. Salt in processed food [online], 2005. Available at http://www.food.gov.uk/healthiereating/salt/ saltmodel. Accessed 18 September 2006.

21 Institute of Grocery Distribution (IGD). Report of the IGD/PIC Industry Nutrition Strategy Group Technical Working Group on Guideline Daily Amounts (GDAs). London: IGD, 2005.

22 Ministry of Agriculture, Fisheries and Food. Food Portion Sizes, 2nd ed. London: HMSO, 1993. 